\title{
Abdominal Obesity and Metabolic Alterations in the Menopausal Transition
}

\author{
Gabriela Berg • Viviana Mesch • Nestor Siseles
}

Published online: 17 March 2012

(C) Springer Science+Business Media, LLC 2012

\begin{abstract}
The prevalence of overweight and obesity is strikingly increasing worldwide. Obesity is a major risk factor for non-insulin-dependent diabetes mellitus, cardiovascular diseases, and cancer, and is also associated with various psychosocial consequences. Menopausal transition is associated with unfavorable changes in body composition and abdominal fat deposition, which is accompanied by an increment of insulin resistance/hyperinsulinemia, a decrease in sex hormone-binding globulin levels and, consequently, an increase in free testosterone and greater androgenicity. Central body fat also correlates with deleterious changes in adipokines and inflammation markers like leptin, C-reactive protein, and adiponectin. In addition, the greater deposition of visceral fat in the menopausal transition contributes to the development of an atherogenic lipoprotein profile, which includes high triglyceride and low high-density lipoprotein (HDL) cholesterol concentrations, as well as an increment of small dense low-density lipoprotein (LDL) subfraction, intermediate density lipoproteins (IDL), apolipoprotein (apo) B/apo A-I ratio and lipoprotein(a).
\end{abstract}

Keywords Abdominal obesity - Menopausal transition . Sex hormones $\cdot$ Inflammation $\cdot$ Lipoproteins

\footnotetext{
G. Berg $(\bowtie) \cdot$ V. Mesch

Clinical Biochemistry Department, Instituto de Fisiopatología y Bioquímica Clínica (INFIBIOC), Faculty of Pharmacy and Biochemistry, University of Buenos Aires, Buenos Aires, Argentina

e-mail: gaberg@ffyb.uba.ar

N. Siseles

Department of Obstetrics and Gynecology, Hospital de Clínicas

"José de San Martín", University of Buenos Aires,

Buenos Aires, Argentina
}

\section{Introduction}

Obesity is the most prevalent nutrition-related disorder in Western countries, and the prevalence of overweight and obesity is increasing worldwide at an alarming rate [1], affecting both developed and developing countries. In the past 20 years, the rates of obesity increased significantly in developing countries that adopted a Western lifestyle, involving decreased physical activity and overconsumption of cheap, energy-dense food. At least 1 billion people worldwide are thought to be overweight (body mass index [BMI] $\left.25-29.9 \mathrm{~kg} / \mathrm{m}^{2}\right)$ or obese $\left(\mathrm{BMI} \geq 30 \mathrm{~kg} / \mathrm{m}^{2}\right)$; at least 300 million people are thought to be obese [1] while one-third of the population is overweight.

The health consequences of obesity are many and varied, ranging from an increased risk of premature death to several nonfatal diseases with adverse impact on quality of life. Obesity is a major risk factor for non-insulin-dependent diabetes mellitus, cardiovascular diseases (CVD) (heart disease, stroke, and hypertension), and cancer [1], and is also associated with various psychosocial consequences.

Although increasing BMI is a powerful predictor of metabolic disease risk, individuals with the same BMI may have greatly different amounts of visceral (central or abdominal) fat, the adipose tissue depot known to be associated with the greatest metabolic risk [2,3]. Abdominal obesity, when accompanied by metabolic derangements, including insulin resistance, low high-density lipoprotein cholesterol (HDL-C), elevated triglycerides (TG), and raised blood pressure, significantly increases the predicted cardiovascular disease risk [4] and constitutes the well-known metabolic syndrome. Insulin resistance and inflammation are the two more common abnormalities that link obesity with adverse metabolic changes $[5,6]$. 
The rapid increases in overweight and obesity are multifactorial and only partly attributable to changes in lifestyle practices [7]. The prevalence of obesity is typically higher in females than males globally. While the reasons for this sex difference in obesity are not clear, fluctuations in female sex hormones at menarche, pregnancy, and menopause may play a role in the adipose tissue expansion. Moreover, weight gain, with an increase in body fat percentage and a concomitant redistribution of fat accumulation from peripheral locations toward increased intra-abdominal depots, is common after the menopause $[8,9]$. This timing suggests that the female sex steroid pattern is important in adipose tissue metabolism and highlights the role of sex hormones on fat distribution, a fact that has been extensively reported $[10,11]$.

The menopause transition begins with the onset of the first menstrual irregularity and ends with cessation of menses [12]. Concerning estradiol levels in this period of life, they are frequently higher than those found in premenopausal women [13, 14], but, indeed, estradiol concentrations in perimenopausal women show a high degree of variability [13]. In turn, changes in androgen levels during the menopausal transition are controversial, although it is generally accepted that testosterone levels do not change significantly in this period of life [15]. Given that alterations of the hormonal profile begin in the early $40 \mathrm{~s}$, in the menopausal transition, consequently, changes in risk factors for CVD (such as anthropometric characteristics) could also be observed in this period. It has been necessary to investigate the behavior of these factors during the transition to menopause and it is still not clear when metabolic alterations occur. Different studies have shown that menopausal transition is associated with unfavorable changes in body composition and abdominal fat deposition $[9,15,16]$.

This review summarizes and discusses the impact of abdominal obesity and the metabolic alterations associated with the menopausal transition.

\section{Sex Hormones and Abdominal Obesity}

\section{Estrogens}

Data in the literature suggest that estrogens play an important role in regulating body fat distribution in women. In a longitudinal study, Lovejoy et al. [17] showed that visceral adipose tissue increased significantly through the menopausal transition from 3-4 years before menopause, and this increment in abdominal fat paralleled the decrease in serum estradiol over time.

Taking into account BMI changes and body fat distribution with declining estrogen levels, several mechanisms have been proposed in relation to alterations in adipose tissue metabolism. It has been described that during the premenopausal period, lipid deposition in gluteofemoral adipocytes is facilitated by lipoprotein lipase (LPL) via estrogen action to assure adequate energy stores for reproduction [18]. This LPL activity in gluteofemoral adipocytes is higher than that of abdominal adipocytes. On the other hand, in postmenopausal women, femoral adipose tissue shows low LPL activity, while in the abdominal region, there is increasing visceral fat deposition and higher lipolytic activity [19]. These changes become evident since the menopausal transition.

Trying to explain how estrogen promotes and maintains the typical female type of fat distribution, Pedersen et al. [20] studied the effects of estradiol in vivo and in vitro on human adipose tissue metabolism and observed that estradiol administration attenuates lipolytic response in subcutaneous abdominal adipocytes, but not in intra-abdominal adipocytes. They found that estradiol directly increases the number of antilipolytic $\alpha$-2A-adrenergic receptors in subcutaneous adipocytes and, in contrast, no effect of estrogen on $\alpha$-2A-adrenergic receptor messenger RNA expression was observed in adipocytes from the visceral fat depots. In other words, estrogen seems to inhibit lipolysis only in subcutaneous depots; consequently, they preferentially stimulate fat storage in subcutaneous versus intra-abdominal fat tissue.

Other authors showed that postmenopausal women under estrogen replacement therapy have significantly lower waist-to-hip ratio [21] and less visceral adipose tissue [22] compared with estrogen non-users.

In a study of women transitioning to menopause, we observed an inverse trend between estradiol levels and waist circumference, a marker of abdominal fat [16]. As it was said above, during menopausal transition, estradiol levels in each woman greatly fluctuate until they decrease completely in postmenopause [13]. This variability could explain why we did not find a strong correlation between this hormone and waist circumference.

\section{Androgens/Testosterone}

There is evidence in the literature that increased testosterone and decreased sex hormone-binding globulin (SHBG) levels are strongly associated with central adiposity. Janssen et al. [23], in a 9-year longitudinal study of a multiethnic cohort undergoing the menopausal transition, showed that bioavailable testosterone, but not estradiol, was strongly related to waist circumference. Furthermore, in multivariate models, the relationship of SHBG to waist circumference was similar to that of bioavailable testosterone. Other authors also found that the major hormonal change associated with central adiposity during the menopausal transition was the increase in the free testosterone index [24], independent of age and estradiol levels. This decrease in SHBG 
and subsequent increase in free testosterone may act directly on fat stores or may modify expression of genes controlling adipocyte differentiation and metabolism.

In a cross-sectional study, our group evaluated the relationship between androgens and cardiovascular risk factors in the menopausal transition [15]. We found an increment in the androgenic milieu that correlated with abdominal fat, insulin-resistance, and atherogenic lipoproteins. According to Janssen et al. [23], the significant correlation between waist circumference and SHBG persisted after a multiple regression analysis with waist circumference as the independent variable.

More recently, Janssen et al. [25••] suggest that testosterone is a strong predictor of visceral fat and, as such, associated with higher cardiovascular risk in women transitioning through menopause. As estradiol production decreases with the proximity of menopause, SHBG levels decline, and thus, the proportion of bioavailable testosterone increases. Dieudonné et al. [26] demonstrated the presence of androgen receptors in the nuclear and cytosolic fractions of human preadipocytes, a decrease of androgen receptor expression during adipogenesis, and an upregulation of these receptors by androgens in vitro. These findings can explain visceral fat accumulation regulated by bioavailable testosterone.

Alternatively, in postmenopause, body mass increment correlates with insulin resistance and hyperinsulinemia, and both situations determine a decrease in SHBG levels and, consequently, an increase in free testosterone and greater androgenicity, all these changes being reflected in higher central adiposity [18] (Fig. 1). In the study of Janssen et al. $[25 \bullet \cdot]$, the association between visceral fat and bioavailable testosterone persisted when both estradiol and insulin resistance were included in the model; therefore, they concluded that the effect of bioavailable testosterone on visceral fat is only partially mediated by insulin resistance.

It has been suggested that these changes begin in the menopausal transition, and therefore, we propose that evaluation of CVD risk in women in this period of life should include androgens, considering abdominal obesity as one of the main determinants of the relation between androgenic parameters and CVD risk factors.

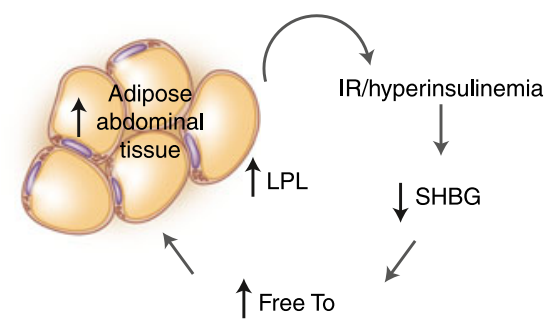

Fig. 1 Relation between central adiposity, insulin resistance (IR) and androgenicity. $L D L$ low-density lipoprotein; $S H B G$ sex hormone-binding globulin
Effect of Hormonal Alterations on Adipokines

\section{Leptin/adiponectin}

Adipose tissue is nowadays considered an important endocrine and paracrine organ that produces several proinflammatory substances [27] that contribute to vascular injury, insulin resistance, and atherogenesis. These adipokines include: tumor necrosis factor (TNF)- $\alpha$, interleukin (IL)-6, leptin, plasminogen activator inhibitor (PAI)-1, angiotensinogen, resistin, and C-reactive protein (CRP). Some of them have a protective role against vascular inflammation and insulin resistance, such as adiponectin and nitric oxide, among others.

Leptin is a 167-amino acid hormone secreted largely by adipose tissue that controls food intake and energy expenditure. Other potential physiological roles for leptin have been described, like its action in the immune and reproductive systems [27].

Leptin concentrations have been reported to be up to four times higher in women than in men [28-30], associated with the higher body fat content observed in women [31]. Estrogen increases leptin concentration [32], which also correlates to adipose distribution and adipocyte size in women but not in men [28, 30]. Different studies have shown that leptin levels increase during the menopausal transition [33, 34], but in postmenopausal women, others found that the increase in leptin levels is associated with aging and not with menopausal status [35]. Furthermore, Hadji et al. [36] showed that neither menopause nor serum estradiol alters plasma leptin concentration, even after controlling for BMI. They found that serum leptin concentrations were significantly higher in pre- and postmenopausal obese women, compared with normal-weight control patients, so only BMI remained as a statistically significant independent predictor for leptin.

Adiponectin belongs to the cytokines secreted by adipose tissue and it is inversely associated with obesity and inflammation. Women present higher circulating levels of adiponectin than men [37]. While some studies showed no differences in adiponectin concentrations between pre- and perimenopausal women [33, 34], others showed them increased in postmenopause [38••]. Sowers et al. [33] reported that mean adjusted adiponectin levels were lower in women during their perimenopausal stage relative to their pre- or postmenopausal stages in both obese and nonobese women, and those differences persisted after adjusting for the covariates.

In reference to most of the adipokines, Ritland et al. [39] observed that centralized body fat was the main contributor to each circulating appetitive hormone in healthy postmenopausal women: androidal-to-gynoidal fat mass ratio (adiponectin), android fat mass (leptin), sagittal abdominal diameter (insulin), or waist circumference (ghrelin) ghrelin. 


\section{Risk Factors Associated with Abdominal Obesity}

Even though most theories consider insulin resistance as the prime factor linking visceral obesity with adverse metabolic changes [40], several studies suggest that other factors like dyslipidemia and chronic systemic inflammation have a main role in vascular disease risk [41, 42].

\section{Insulin Resistance/Hyperinsulinemia}

Abdominal obesity is a highly prevalent feature of the insulin resistant state; however, the mechanisms by which they are related are not fully understood. Analysis of abdominal obesity by imaging studies have generally reached the conclusion that it is the excess of intra-abdominal or visceral adipose tissue and not the amount of subcutaneous abdominal fat which is the key correlate of the metabolic abnormalities observed in overweight/obese patients [43]. Multiple environmental and genetic factors are thought to influence the manifestation of abdominal obesity. Intraabdominal fat increases with age in both overweight and normal-weight individuals, independently of changes in total body fat [44]. In premenopausal women, Ross et al. [45] have observed that individuals matched for subcutaneous abdominal adiposity, but with either a low versus a high accumulation of visceral adipose tissue, were markedly different in their levels of insulin resistance and glucose tolerance. However, after being matched for visceral adiposity, individuals with low or high levels of subcutaneous fat were found not to differ in insulin sensitivity [45].

The expanded adipose tissue contributes to expose the liver to high concentrations of free fatty acids (FFA), impairing several hepatic metabolic processes leading to hyperinsulinemia [4]. On the other side, there is an increase in the secretion of different adipokines, such as IL- 6 and TNF- $\alpha$, which also contributes to the insulin-resistant state.

The World Health Organization MONICA (Multinational Monitoring of Trends and Determinants in Cardiovascular Disease) Project, monitoring CVD in 21 countries, suggested that the relationship between insulin concentration and coronary heart disease was potentially stronger in women than in men [46]. In addition, obesity development is accelerated after menopause; factors such as loss of estrogens, the aging process, and changes in lifestyle all may be contributors $[47,48]$.

In fact, the increase in insulin resistance observed in postmenopause by many authors [49] could be explained not only by the menopause effect, but also by aging and abdominal obesity $[8,50]$. Given the difficulties in the assessment of insulin resistance by the standard methods, for epidemiologic and clinical studies, more simple and indirect methods have been advocated [9]. The frequency of insulin resistance increases from the time of the menopausal transition to the postmenopause. A study of ours [9] showed that the prevalence of the metabolic syndrome rises from the earlier stages of the menopausal transition, without differences with postmenopausal women, while it was not detected in the premenopausal group.

\section{Dyslipidemia}

Some authors have observed that age and menopause both can influence the changes in body fat distribution $[9,51]$ and that, in turn, central obesity contributes to the impairment of the lipoprotein profile.

High visceral adipose tissue accumulation has been associated with a deteriorated metabolic profile such as high TG and low HDL-C concentrations in postmenopausal women $[8,9,16]$. The association between visceral adipose tissue accumulation and deteriorated metabolic profile may be explained in part by high lipolytic action of visceral adipose tissue, leading to an increased flux of FFA in portal circulation to liver that increases intracellular availability of TG and stimulates the assembly and secretion of very lowdensity lipoprotein (VLDL) particles. Moreover, in postmenopausal women (PMW), an adverse lipoprotein pattern has been described, even though HDL-C levels tend to be maintained or slightly decreased $[16,52]$. In turn, hepatic lipase is a key enzyme involved in the hydrolysis of TG and phospholipids contained in low-density lipoprotein (LDL) and HDL particles, and, in cooperation with cholesteryl ester transfer protein (CETP), it also contributes to lipoprotein remodeling. We previously reported a high hepatic lipase activity in menopause [53], likely associated with the fall of endogenous estrogens. A consequence of higher hepatic lipase activity may be the increase of small dense LDL subfraction, already described in PMW [8, 54]. This particle per se has a greater atherogenic potential than other LDL subclasses [55].

The high proportion of this atherogenic subfraction leads to an enhanced passage of these particles through the endothelium allowing their binding to proteoglycans, given that small dense LDL have greater affinity than large buoyant LDL [55]. Results from studies on women with clinical and subclinical disease showed that small dense LDL was related to high incidence of coronary heart disease among older women [56]. It can be suggested that the higher small dense LDL proportion is the higher LDL oxidability. Moreover, the higher abdominal fat accumulation measured as waist circumference is the higher LDL oxidability. Therefore, women with high degree of insulin resistance would present an increase in small dense LDL proportion and LDL susceptibility to oxidation [52].

In reference to the presence of remnants of lipoproteins, we have described an increase in intermediate-density lipoproteins (IDL) since the menopausal transition [16] as well 
as in PMW [53]. These remnants are highly associated with CVD, especially in women [57]. Given their small size, they can cross the endothelium, and in the subendothelium are considered modified lipoproteins and are phagocytosed by macrophages [58]. The increase in remnants of lipoproteins has been highly associated with abdominal obesity [50].

Apolipoprotein (apo) B and the apo B/apo A-I ratio also has been described to be increased in PMW. The former is considered a useful risk marker with high predictive value because each atherogenic lipoprotein particle contains only one copy of apo B [59]. The ratio of apo B/apo A-I reflects the balance between pro- and antiatherogenic lipoproteins through their structural apolipoproteins, and this ratio has been demonstrated to be a strong predictor of acute myocardial infarction in men and women [60]. This ratio is also increased in PMW and correlates with the higher abdominal obesity [52].

Another modified lipoprotein that is also increased since the menopausal transition is Lipoprotein(a), or Lp(a) [16], which is greatly associated with CVD in women [61]. Estrogens have been shown to decrease $L p(a)$ synthesis, and the decrease in estrogens since the late menopausal transition would condition the increase in this lipoprotein (Fig. 2).

\section{Inflammation}

An important issue in understanding obesity is the concept that it is characterized by a state of chronic low-grade inflammation [62]. While the general assumption is that inflammation is a consequence of obesity, it has been suggested that obesity, in fact, is a result of inflammatory disease [63]. Adipose tissue secretes factors that stimulate

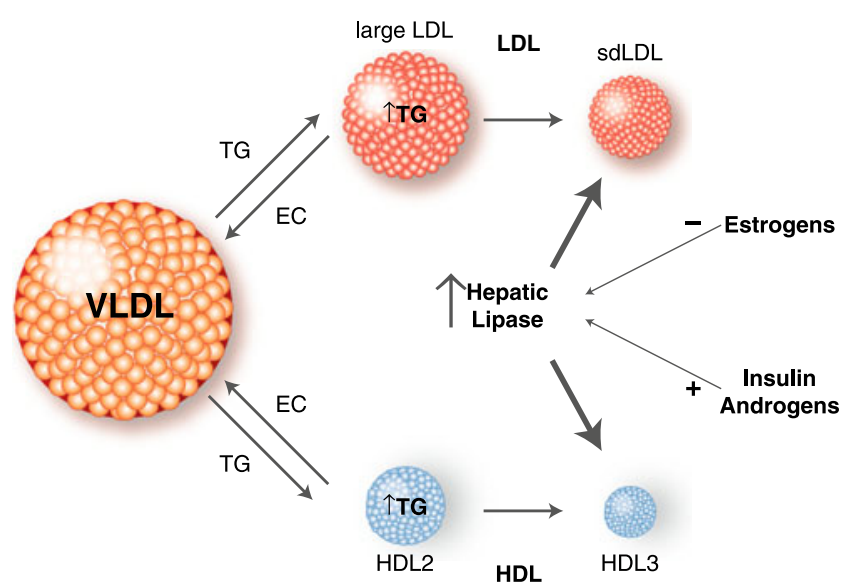

Fig. 2 Hepatic lipase behavior in postmenopause and insulinresistance conditions. After the increase in triglyceride (TG) levels, low- (LDL) and high-density lipoprotein (HDL) are enriched in TG and are better substrates for hepatic lipase. In postmenopause, there is an increase in the activity of hepatic lipase, promoting the predominance of small and dense LDL and of smaller HDL. VLDL very low-density lipoprotein; $E C$ esterified cholesterol; $s d L D L$ small dense low-density lipoprotein the production of inflammatory markers from the liver and other organs, and the adipocytes themselves are the immediate source of most of these inflammatory markers [62]. Visceral adipose tissue from obese patients also has been found to secrete higher levels of PAI-1, IL-6, TNF- $\alpha$, and leptin and lower levels of adiponectin compared with lean patients [64-66]. Circulating inflammatory markers elevated in obese patients include CRP and serum amyloid A (SAA) [67, 68].

In vitro and animal studies indicate that CRP may actively participate in plaque development by promoting endothelial dysfunction, monocyte adhesion to the endothelium, macrophage cholesterol accumulation, and fibrin breakdown (reduced plaque stability) increasing cardiovascular risk [69].

The increase in visceral adiposity across the menopausal transition may result in the development of proinflammatory adipokine changes. Lee et al. [38••] studied 69 healthy women who were followed up longitudinally from premenopausal to postmenopausal status. They found that SAA, monocyte-chemotactic protein-1 (MCP-1), tissue plasminogen activator antigen (tPA), and adiponectin levels increased significantly between the two measurement occasions. The increase in intra-abdominal fat was positively correlated with changes in leptin, tPA, SAA, and CRP levels and negatively correlated with the change in adiponectin. These findings suggest that the increase in visceral adiposity across the menopausal transition results in adverse effects on adipokine and inflammation levels.

We have previously discussed the behavior of leptin and adiponectin (this last one considered today as an inflammatory marker itself) in perimenopausal women and PMW. However, the most common and accepted inflammatory marker is CRP. Perry et al. [70] observed that androidal fat mass contributed to CRP and fibrinogen [70], but showed no relationship to cytokines. This may suggest that, in healthy PMW, acute-phase proteins may be used as earlier indicators of CVD risk than inflammatory cytokines.

Studies by Miksztowicz et al. [71] and Muzzio et al. [72••] revealed higher levels of high-sensitivity CRP in premenopausal women and PMW with metabolic syndrome and in healthy PMW, respectively, and this biomarker was associated with other risk factors like metalloproteinases and small dense LDL. Lee et al. [38••] reported that CRP, IL-6, and TNF- $\alpha$ did not change across the menopausal transition; however, CRP directly correlated with abdominal fat.

\section{Conclusions}

Different studies have shown that menopausal transition is associated with unfavorable changes in body composition 
and abdominal fat deposition. The conjunction of higher free testosterone levels, lower SHBG, and an increase in insulin resistance contributes to an increment of visceral fat. This increase in abdominal adiposity across the menopausal transition is also associated to an unfavorable lipoprotein profile and also may result in adverse effects on adipokines and inflammation levels.

Women transitioning to menopause should consider adopting beneficial changes in lifestyle to prevent the development of abdominal obesity and, consequently, type 2 diabetes and CVD.

Disclosures No potential conflicts of interest relevant to this article were reported.

\section{References}

Papers of particular interest, published recently, have been highlighted as:

•- Of major importance

1. World Health Organization (2008) Global strategy on diet, physical activity and health. http://www.who.int/dietphysicalactivity/ publications/facts/obesity/en/. Accessed May 15, 2008

2. Nieves DJ, Cnop M, Retzlaff B, Walden CE, Brunzell JD, Knopp $\mathrm{RH}, \mathrm{Kahn}$ SE. The atherogenic lipoprotein profile associated with obesity and insulin resistance is largely attributable to intraabdominal fat. Diabetes. 2003;52:172-9.

3. Abate N, Garg A. Heterogeneity in adipose tissue metabolism: causes, implications and management of regional adiposity. Prog Lipid Res. 1995;34:53-70.

4. Lakka HM, Laaksonen DE, Lakka TA, Niskanen LK, Kumpusalo E, Tuomilehto J, Salonen JT. The metabolic syndrome and total and cardiovascular disease mortality in middle-aged men. JAMA. 2002;288:2709-16.

5. Reaven GM, Chen YD. Role of insulin in regulation of lipoprotein metabolism in diabetes. Diabetes Metab Rev. 1988;4:639-52.

6. Festa A, D'Agostino Jr R, Howard G, Mykkänen L, Tracy RP, Haffner SM. Chronic subclinical inflammation as part of the insulin resistance syndrome: the Insulin Resistance Atherosclerosis Study (IRAS). Circulation. 2000;102:42-7.

7. Bray GA, Bellanger T. Epidemiology, trends, and morbidities of obesity and the metabolic syndrome. Endocrine. 2006;29:109-17.

8. Carr M. The emergence of the metabolic syndrome with menopause. J Clin Endocrinol Metab. 2003;88:2404-11.

9. Mesch VR, Boero LE, Siseles NO, Royer M, Prada M, Sayegh F, Schreier L, Benencia HJ, Berg GA. Metabolic syndrome throughout the menopausal transition: influence of age and menopausal status. Climacteric. 2006;9:40-8.

10. Haarbo J, Marslew U, Gotfredsen A, Christiansen C. Postmenopausal hormone replacement therapy prevents central distribution of body fat after menopause. Metabolism. 1991;40:1323-6.

11. Tchernof A, Poehlman ET, Després JP. Body fat distribution, the menopause transition and hormone replacement therapy. Diabetes Metab. 2000;26:12-20.

12. McKinlay SM, Brambilla DJ, Posner JG. The normal menopausal transition. Maturitas. 1992;14:103-15.

13. Burger HG, Dudley EC, Robertson DM, Dennerstein L. Hormonal changes in the menopause transition. Recent Prog Horm Res. 2002;57:257-75.
14. Hall JE. Neuroendocrine physiology of the early and late menopause. Endocrinol Metab Clin North Am. 2004;33:637-59.

15. Mesch VR, Siseles NO, Maidana PN, Boero LE, Sayegh F, Prada M, Royer M, Schreier L, Benencia HJ, Berg GA. Androgens in relationship to cardiovascular risk factors in the menopausal transition. Climacteric. 2008;11:509-17.

16. Berg G, Mesch V, Boero L, Sayegh F, Prada M, Royer M, Muzzio ML, Schreier L, Siseles N, Benencia H. Lipid and lipoprotein profile in menopausal transition. Effects of hormones, age and fat distribution. Horm Metab Res. 2004;36:215-20.

17. Lovejoy JC, Champagne CM, de Jonge L, Xie H, Smith SR. Increased visceral fat and decreased energy expenditure during the menopausal transition. Int J Obes (Lond). 2008;32:949-58.

18. Sowers M, Tisch J. Insulin resistance, body weight, obesity, body composition, and the menopausal transition. In: Lobo R, Kelsey J, Marcus R, editors. Menopause, biology and pathobiology. San Diego: Academic; 2000. p. 245-60.

19. Björntorp P. The regulation of adipose tissue distribution in humans. Int J Obes Relat Metab Disord. 1996;20:291-302.

20. Pedersen SB, Kristensen K, Hermann PA, Katzenellenbogen JA, Richelsen B. Estrogen controls lipolysis by up-regulating $\alpha 2 \mathrm{~A}-$ adrenergic receptors directly in human adipose tissue through the estrogen receptor $\alpha$. Implications for the female fat distribution. $\mathrm{J}$ Clin Endocrinol Metab. 2004;89:1869-78.

21. Espeland MA, Stefanick ML, Kritz-Silverstein D, Fineberg SW, Waclawiw MA, James MK, et al. Effect of postmenopausal hormone therapy on body weight and waist and hip girths. J Clin Endocrinol Metab. 1997;82:1549-56.

22. Munoz J, Derstine A, Gower BA. Fat distribution and insulin sensitivity in postmenopausal women: influence of hormone replacement. Obes Res. 2002;10:424-31.

23. Janssen I, Powell LH, Crawford S, Lasley B, Sutton-Tyrrell K. Menopause and the metabolic syndrome: the Study of Women's Health Across the Nation. Arch Intern Med. 2008;168:1568-75.

24. Guthrie JR, Dennerstein L, Taffe JR, et al. Central abdominal fat and endogenous hormones during the menopausal transition. Fertil Steril. 2003;79:1335-40.

25. •• Janssen I, Powell LH, Kazlauskaite R, Dugan SA. Testosterone and visceral fat in midlife women: the Study of Women's Health Across the Nation (SWAN) fat patterning study. Obesity (Silver Spring). 2010;18:604-10. This study showed that in women transitioning to menopause, bioavailable testosterone was associated with visceral fat, independent of age, race, percent total body fat, and other cardiovascular risk factors.

26. Dieudonné MN, Pecquery R, Boumediene A, Leneveu MC, Giudicelli Y. Androgen receptors in human preadipocytes and adipocytes: regional specificities and regulation by sex steroids. Am J Physiol. 1998;274:C1645-52.

27. Gomes F, Telo DF, Souza HP, Nicolau JC, Halpern A, Serrano Jr $\mathrm{CV}$. Obesity and coronary artery disease: role of vascular inflammation. Arq Bras Cardiol. 2010;94:255-61.

28. Garaulet M, Perex-Llamas F, Fuente T, et al. Anthropometric, computed tomography and fat cell data in an obese population: relationship with insulin, leptin, tumor necrosis factor-alpha, sex hormonebinding globulin and sex hormones. Eur J Endocrinol. 2000;143:657-66.

29. Gottschling-Zeller H, Birgel M, Scriba D, et al. Depot-specific release of leptin from subcutaneous and omental adipocytes in suspension culture: Effect of tumor necrosis factor-alpha and transforming growth factor-beta1. Eur Endocrinol. 1999;141: 436-42.

30. Havel PJ, Kasim-Karakas S, Dubuc GR, et al. Gender differences in plasma leptin concentrations. Nat Med. 1996;2:949-50.

31. Perseghin G, Scifo P, Pagliato E, et al. Gender factors affect fatty acids-induced insulin resistance in nonobese humans: effects of oral steroidal contraception. Clin Endocrinol Metab. 2001;86:3188-96. 
32. Margetic S, Gazzola C, Pegg GG, Hill RA. Leptin: a review of its peripheral actions and interactions. Obes Relat Metab Disord. 2002;26:1407-33.

33. Sowers MR, Wildman RP, Mancuso P, Eyvazzadeh AD, Karvonen-Gutierrez CA, Rillamas-Sun E, Jannausch ML. Change in adipocytokines and ghrelin with menopause. Maturitas. 2008;59:149-57.

34. Wildman RP, Mancuso P, Wang C, Kim M, Scherer PE, Sowers MR. Adipocytokine and ghrelin levels in relation to cardiovascular disease risk factors in women at midlife: longitudinal associations. Int J Obes (Lond). 2008:32:740-8.

35. Sherk VD, Malone SP, Bemben MG, Knehans AW, Palmer IJ, Bemben DA. Leptin, fat mass, and bone mineral density in healthy pre- and postmenopausal women. J Clin Densitom. 2011;14:321-5.

36. Hadji P, Hars O, Bock K, Sturm G, Bauer T, Emons G, Schulz KD. The influence of menopause and body mass index on serum leptin concentrations. Eur J Endocrinol. 2000;143:55-60.

37. Scherer PE. Adipose tissue: from lipid storage com partment to endocrine organ. Diabetes. 2006;55:1537-45.

38. •• Lee CG, Carr MC, Murdoch SJ, Mitchell E, Woods NF, Wener $\mathrm{MH}$, Chandler WL, Boyko EJ, Brunzell JD. Adipokines, inflammation, and visceral adiposity across the menopausal transition: a prospective study. J Clin Endocrinol Metab. 2009;94:1104-10. This study showed that women going during the menopausal transition have deleterious changes in inflammatory markers and adipokines that correlate with increased visceral adiposity.

39. Ritland LM, Alekel DL, Matvienko OA, Hanson KB, Stewart JW, Hanson LN, Reddy MB, Van Loan MD, Genschel U. Centrally located body fat is related to appetitive hormones in healthy postmenopausal women. Eur J Endocrinol. 2008;158:889-97.

40. Reaven GM. Banting lecture 1988. Role of insulin resistance in human disease. Diabetes. 1988;37:1595-607.

41. Ross R. Atherosclerosis is an inflammatory disease. Am Heart J. 1999;138(5 Pt 2):S419-20.

42. Grundy SM. Cholesterol and coronary heart disease. The 21st century. Arch Intern Med. 1997;157:1177-84.

43. Després JP, Lemieux I, Bergeron J, Pibarot P, Mathieu P, Larose E, Rodés-Cabau J, Bertrand OF, Poirier P. Abdominal obesity and the metabolic syndrome: contribution to global cardiometabolic risk. Arterioscler Thromb Vasc Biol. 2008;28:1039-49.

44. Carr M, Brunzell J. Abdominal obesity and dyslipidemia in the metabolic syndrome: importance of type 2 diabetes and familial combined hyperlipidemia in coronary artery disease risk. J Clin Endocrinol Metab. 2004;89:2601-7.

45. Ross R, Freeman J, Hudson R, Janssen I. Abdominal obesity, muscle composition, and insulin resistance in premenopausal women. J Clin Endocrinol Metab. 2002;87:5044-51.

46. Eliasson M, Asplund K, Evrin PE, Lindahl B, Lundblad D. Hyperinsulinemia predicts low tissue plasminogen activator activity in a healthy population: the Northern Sweden MONICA Study. Metabolism. 1994;43:1579-86.

47. Shi H, Clegg DJ. Sex differences in the regulation of body weight. Physiol Behav. 2009;97:199-204.

48. Barton M. Obesity and aging: determinants of endothelial cell dysfunction and atherosclerosis. Pflugers Arch. 2010;460:825-37.

49. Munoz J, Derstine A, Gower B. Fat distribution and insulin sensitivity in postmenopausal women: influence of hormone replacement. Obes Res. 2002;10:424-31.

50. Pascot A, Despres JP, Lemieux I, et al. Deterioration of the metabolic risk profile in women. Respective contributions of impaired glucose tolerance and visceral fat accumulation. Diabetes Care. 2001;24:902-8.

51. Chang $\mathrm{C}, \mathrm{Wu} \mathrm{C}$, Yao W, et al. Relationships of age, menopause and central obesity on cardiovascular disease risk factors in Chinese women. Int J Obes. 2000;24:1699-704.
52. Muzzio ML, Berg G, Zago V, Basilio F, Sanguinetti S, Lopez G, Brites F, Wikinski R, Schreier L. Circulating small dense LDL, endothelial injuring factors and fibronectin in healthy postmenopausal women. Clin Chim Acta. 2007;381:157-63.

53. Berg G, Siseles N, González AI, Contreras Ortiz O, Tempone A, Wikinski R. Higher values of hepatic lipase activity in postmenopause: relationship with atherogenic intermediate density and low density lipoproteins. Menopause. 2001;8:51-7.

54. Berg G, Muzzio ML, Wikinski R, Schreier L. A new approach to the quantitative measurement of dense LDL subfractions. Nutr Metab Cardiovasc Dis. 2004;14:73-80.

55. Berneis KK, Krauss RM. Metabolic origins and clinical significance of LDL heterogeneity. J Lipid Res. 2002;43:1363-79.

56. Kuller L, Arnold A, Tracy R, et al. Nuclearmagnetic resonance spectroscopy of lipoproteins and risk of coronary heart disease in the cardiovascular health study. Arterioscler Thromb Vasc Biol. $2000 ; 1: 1175-80$.

57. McNamara JR, Shah PK, Nakajima K, Cupples LA, Wilson PW, Ordovas JM, Schaefer EJ. Remnant-like particle (RLP) cholesterol is an independent cardiovascular disease risk factor in women: results from the Framingham Heart Study. Atherosclerosis. 2001;154:229-36.

58. Nordestgaard BG, Agerholm-Larsen B, Mortensen A, et al. Intermediate density lipoprotein cholesterol as the best lipoprotein predictor of atherosclerosis severity in the Watanabe heritable hyperlipidemic rabbit. Atherosclerosis. 1997;132:119-22.

59. Sniderman AD. How, when, and why to use apolipoprotein B in clinical practice. Am J Cardiol. 2002;90:48i-54i.

60. Walldius G, Jungner I. The apoB/apoA-I ratio: a strong, new risk factor for cardiovascular disease and a target for lipid-lowering therapy - a review of the evidence. J Intern Med. 2006;259:493519.

61. Sposito AC, Mansur AP, Maranhão RC, Martinez TR, Aldrighi JM, Ramires JA. Triglyceride and lipoprotein (a) are markers of coronary artery disease severity among postmenopausal women. Maturitas. 2001;39:203-8.

62. Calabro P, Yeh ET. Obesity, inflammation, and vascular disease: the role of the adipose tissue as an endocrine organ. Subcell Biochem. 2007;42:63-91.

63. Das UN. Is obesity an inflammatory condition? Nutrition. 2001;17:953-66.

64. He G, Pedersen SB, Bruun JM, Lihn AS, Jensen PF, Richelsen B. Differences in plasminogen activator inhibitor 1 in subcutaneous versus omental adipose tissue in non-obese and obese subjects. Horm Metab Res. 2003;35:178-82.

65. Winkler G, Kiss S, Keszthelyi L, Sapi Z, Ory I, Salamon F, Kovacs M, Vargha P, Szekeres O, Speer G, Karadi I, Sikter M, Kaszas E, Dworak O, Gero G, Cseh K. Expression of tumor necrosis factor (TNF)- $\alpha$ protein in the subcutaneous and visceral adipose tissue in correlation with adipocyte cell volume, serum TNF- $\alpha$, soluble serum TNF-receptor-2 concentrations and C-peptide level. Eur J Endocrinol. 2003;149:129-35.

66. Maury E, Ehala-Aleksejev K, Guiot Y, Detry R, Vandenhooft A, Brichard SM. Adipokines oversecreted by omental adipose tissue in human obesity. Am J Physiol Endocrinol Metab. 2007;293: E656-65.

67. Yang RZ, Lee MJ, Hu H, Pollin TI, Ryan AS, Nicklas BJ, Snitker S, Horenstein RB, Hull K, Goldberg NH, Goldberg AP, Shuldiner AR, Fried SK, Gong DW. Acute-phase serum amyloid A: an inflammatory adipokine and potential link between obesity and its metabolic complications. PLoS Med. 2006;3:e287.

68. Bahceci M, Gokalp D, Bahceci S, Tuzcu A, Atmaca S, Arikan S. The correlation between adiposity and adiponectin, tumor necrosis factor alpha, interleukin- 6 and high sensitivity C-reactive protein levels. Is adipocyte size associated with inflammation in adults? J Endocrinol Invest. 2007;30:210-4. 
69. Corcoran MP, Meydani M, Lichtenstein AH, Schaefer EJ, Dillard A, Lamon-Fava S. Sex hormone modulation of proinflammatory cytokine and C-reactive protein expression in macrophages from older men and postmenopausal women. J Endocrinol. 2010;206:217-24.

70. Perry CD, Alekel DL, Ritland LM, Bhupathiraju SN, Stewart JW, Hanson LN, Matvienko OA, Kohut ML, Reddy MB, Van Loan $\mathrm{MD}$, Genschel U. Centrally located body fat is related to inflammatory markers in healthy postmenopausal women. Menopause. 2008;15:619-27.
71. Miksztowicz V, Muzzio ML, Royer M, Prada M, Wikinski R, Schreier L, Berg G. Increased plasma activity of metalloproteinase 2 in women with metabolic syndrome. Metabolism. 2008;57:1493-6.

72. •- Muzzio ML, Miksztowicz V, Repetto EM, Brites F, Berg G, Schreier L. Increased MMP-2 in healthy postmenopausal women. Ann Clin Biochem. 2012;49:75-9. This study showed that in postmenopausal women, there is an increase in MMPs activity and that this increase correlates with the presence of adhesion molecules. 